\title{
CALCUL DES CONTRAINTES DANS UNE COUCHE MINCE DEPOSEE SUR UN SUBSTRAT
}

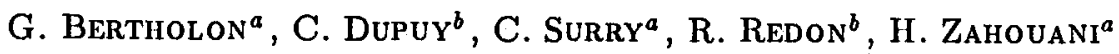 \\ a ENISE, 58 rue Jean Parot, Saint-Etienne 42023 cedex, France \\ ${ }^{b}$ Université Claude Bernard-Lyon 1, Villeurbanne, 69622 cedex, France
}

\begin{abstract}
CALCULATIONS OF THE CONSTRAINTS IN A THIN FILM DEPOSITED ON TIIE SUBSTRATE: The aim of the present paper is to give simple calculations of the constraints in a thin film deposited on the substrate. The thin film can be obtained by the recrystallization of the surface. It is considered in relation to its substrate, where the force is linearly proportional to the surface area of the film. On the other hand, it is considered to be under the action of a force related to the thermic and compressional constraints. The initial and final boundary conditions in the linearized model play an essential role in the model adopted.
\end{abstract}

PACS numbers: 68.55.-a

\section{Introduction}

On étudie le calcul des moments de flexion et des flèches d'une poutre ou d'une plaque mince soumise à une précontrainte $\boldsymbol{P}$ de type thermique par exemple, soumise de la part du substrat sur lequel la poutre est posée, à une réaction proportionnelle à la flèche. On note $\boldsymbol{E}$ le module de Young et $\boldsymbol{I}-$ l'inertie de la barre. Cette poutre est chargée à une densité d'effort extérieur $f(x)$ :

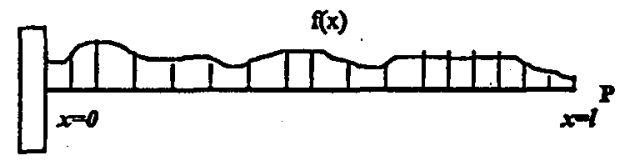

L'équation liant la fléche $u(x)$ aux forces extérieures dans le cadre d'une théorie linéarisée est

$$
\begin{aligned}
& \frac{\mathrm{d}^{2}}{\mathrm{~d} x^{2}}\left(E I \frac{\mathrm{d}^{2} u}{\mathrm{~d} x^{2}}\right)+\alpha u+P \frac{\mathrm{d}^{2} u}{\mathrm{~d} x^{2}}=f(x), \\
& u(0)=u(l)=0, \quad u^{\prime}(0)=u^{\prime}(l)=0 .
\end{aligned}
$$


Les méthodes de calcul classiques utilisent des éléments de poutre prenant comme degré de liberté les flèches et les rotations de noeuds en prenant comme interpolée des cubiques. Cette méthode peut être vue comme une méthode de fonctions splines, mais donne des moments qui sont linéaires par élément et continus aux noeuds de la discrétisation.

Nous proposons une méthode plus simple utilisant des interpolations linéaires pour les moments et les flèches de type mixte. Cette méthode a l'avantage d'être plus simple à programmer, ne faisant appara ître que des matrices tridiagonales.

Elle a d'autre part une signification mécanique plus adaptée car elle sépare l'équilibre de la structure de la loi de comportement, toutes deux écrites sous forme globale [1].

\section{Formulation variationnelle du probleme}

\subsection{Méthodes aux déplacements}

Posons $\Omega=] 0,1[$ :

$\mathcal{H}_{2}^{0}(\Omega)=\left\{v(x) / \int_{0}^{l} E I\left(\frac{\mathrm{d}^{2} v}{\mathrm{~d} x^{2}}\right) \mathrm{d} x<+\infty ; v(0)=v^{\prime}(0)=v(l)=v^{\prime}(l)=0\right\}$

avec

$$
\|v(x)\|_{\mathcal{F}_{2}^{0}(\Omega)}^{2}=\int_{0}^{2} E I\left(\frac{\mathrm{d}^{2} v}{\mathrm{~d} x^{2}}\right)^{2} \mathrm{~d} x .
$$

La solution des Eqs. (1) et (2) est un minimum de $J(v)$ sur $\mathcal{H}_{2}^{0}$ :

$$
J(v)=\frac{1}{2} a(v, v)+\frac{\alpha}{2} \int_{0}^{l} v^{2} \mathrm{~d} x-\frac{P}{2} \int_{0}^{1}\left(\frac{\mathrm{d} v}{\mathrm{~d} x}\right)^{2} \mathrm{~d} x-L(v)
$$

avec

$$
a(v, v)=\int_{0}^{l} E I\left(v^{\prime \prime}\right)^{2} \mathrm{~d} x \quad \text { et } \quad L(v)=\int_{0}^{l} f v \mathrm{~d} x .
$$

\subsection{Méthode mixte}

Avant de rentrer dans un formalisme mathématique rigoureux, l'idée est de remplacer l'équation d'ordre 4 par un systéme d'équations d'ordre 2:

Posons

$$
M=E I \frac{\mathrm{d}^{2} u}{\mathrm{~d} x^{2}} .
$$

$$
\begin{aligned}
& \frac{\mathrm{d}^{2}}{\mathrm{~d} x^{2}} M(x)+\alpha u+P \frac{\mathrm{d}^{2} u}{\mathrm{~d} x^{2}}=f(x), \\
& u(0)=u^{\prime}(0)=u(l)=u^{\prime}(l)=0 .
\end{aligned}
$$

Définissons

$$
\mathcal{U}(x)=\left\{v(x) / \int_{0}^{l} v^{\prime 2} \mathrm{~d} x<+\infty ; \quad v(0)=v(l)=0\right\}=\mathcal{H}_{1}^{0}(\Omega)
$$




$$
\|v\|_{\mathcal{U}}^{2}=\int_{0}^{l}{v^{\prime}}^{2} \mathrm{~d} x
$$

Nous aurons

$$
\frac{1}{2} a(v, v)=\sup _{\bar{M} \in L^{2}(\Omega)}\left\{b(\bar{M}, v)-\frac{1}{2} a^{*}(\bar{M}, \bar{M})\right\}
$$

avec

$$
b(\bar{M}, v)=\int_{0}^{l} \bar{M} \frac{\mathrm{d}^{2} v}{\mathrm{~d} x^{2}} \mathrm{~d} x \quad \text { et } \quad a^{*}(\bar{M}, \bar{M})=\frac{1}{2} \int_{0}^{l} \frac{\bar{M}^{2}}{E I} \mathrm{~d} x .
$$

En utilisant la densité de $\mathcal{H}_{0}^{2}(\Omega) \subset \mathcal{H}_{0}^{1}(\Omega) \subset L(\Omega)$ dans $(7)$, on a

$$
b(\bar{M}, v)=\int_{0}^{l} \bar{M} \frac{\mathrm{d}^{2} v}{\mathrm{~d} x^{2}} \mathrm{~d} x=-\int_{0}^{l} \frac{\mathrm{d} \bar{M}}{\mathrm{~d} x} \frac{\mathrm{d} v}{\mathrm{~d} x} \mathrm{~d} x \quad \forall v \in \mathcal{H}_{0}^{1}(\Omega) \quad \text { et } \quad \forall \bar{M} \in \mathcal{H}^{1}(\Omega) .
$$

On posera alors

$$
\begin{aligned}
& \bar{b}(\bar{M}, \bar{v})=-\int_{0}^{l} \frac{\mathrm{d} \bar{M}}{\mathrm{~d} x} \frac{\mathrm{d} \bar{v}}{\mathrm{~d} x} \mathrm{~d} x, \\
& \forall \bar{M} \in L^{2}(\Omega) \quad a^{*}(\bar{M}, M)-b(\bar{M}, u)=0, \\
& \forall v \in \mathcal{H}_{0}^{2}(\Omega) \quad b(M, v)-P \int_{0}^{l} u^{\prime} v^{\prime} \mathrm{d} x+\alpha \int_{0}^{l} u v \mathrm{~d} x=L(v),
\end{aligned}
$$

et la formulation mixte modifiée s'écrit

$$
\begin{aligned}
& \forall \bar{M} \in \mathcal{H}^{1}(\Omega) \quad a^{*}(\bar{M}, M)-\bar{b}(\bar{M}, u)=0, \\
& \forall v \in \mathcal{H}_{0}^{1}(\Omega) \quad b(M, v)-P \int_{0}^{l} u^{\prime} v^{\prime} \mathrm{d} x+\alpha \int_{0}^{l} u v \mathrm{~d} x=L(v) .
\end{aligned}
$$

Le passage de $\{(8),(9)\}$ à $\{(10),(11)\}$ se fait en utilisant des arguments de dualité et de densité évoqués ci-dessus. Equation (8) exprime la loi de comportement globale, comme (10); Eqs. (9) et (11) traduisent l'équilibre global de la structure considéré.

On doit cependant noter que, si on considère l'ensemble

$$
Z=\left\{\bar{M} / \forall v \in \mathcal{H}_{0}^{1}(\Omega) \quad \bar{b}(\bar{M}, v)=0\right\} .
$$

$\mathrm{Z}$ est l'ensemble des moments de flexion qui sont linéaires sur $\Omega$. L'ensemble des moments autoéquilibrés vérifiant $\mathrm{d}^{2} M / \mathrm{d} x^{2}=0$ est un espace vectoriel de dimension 2 correspondant au degré d'hyperstaticité de la barre étudiée.

En prenant $\bar{M}=v \in \mathcal{H}_{0}^{1}(\Omega) \subset \mathcal{H}^{1}(\Omega)$, on voit que la condition de Brezzi

$$
\sup _{\bar{M} \in \mathcal{H}^{1}(\Omega)} \frac{b(\bar{M}, v)}{\|v\|} \geq C\|v\|_{\mathcal{H}_{0}^{1}(\Omega)}
$$

est satisfaite, assurant l'unicité du problème étudié. 


\section{Méthode d'approximations}

Prenons deux espaces de dimension finie $\Sigma_{h}$ et $U_{h}$ tels que $\Sigma_{h} \subset \mathcal{H}^{1}(\Omega)$ et $U_{h} \subset \mathcal{H}_{0}^{1}(\Omega): \Sigma_{h}=\left\{\bar{M}_{h} \in \mathcal{H}^{1}(\Omega) / \bar{M}_{h} \in P_{1}\left(K_{e}\right) \cap C^{0}(\bar{\Omega}) \quad \forall e 1 \leq e \leq N\right\}$ avec $U_{h}=\left\{v_{h} \in \mathcal{H}_{0}^{1}(\Omega) / v_{h} \in P_{1}\left(K_{e}\right) \cap C^{0}(\Omega) \quad \forall e 1 \leq e \leq N\right\} . N$ est le nombre d'éléments pour $\Omega=] 0,1[$.

Une fois discrétisé, le problème $\{(10),(11)\}$ s'écrit

$\forall \overline{M_{h}} \in \Sigma_{h} \quad a^{*}\left(\overline{M_{h}}, M_{h}\right)-\bar{b}\left(\overline{M_{h}}, u_{h}\right)=0$,

$\forall v_{h} \in U_{h} \quad b\left(M_{h}, v_{h}\right)-\int_{0}^{l} P u_{h}^{\prime} v_{h}^{\prime} \mathrm{d} x+\alpha \int_{0}^{l} u_{h} v_{h} \mathrm{~d} x=\int_{0}^{l} f v_{h} \mathrm{~d} x$.

$\left(M_{h}, u_{h}\right)$ est la solution du problème discrétisé.

On a l'estimation d'erreur

$\left\|u-u_{h}\right\|_{\mathcal{H}_{0}^{1}(\Omega)}+\left\|M-M_{h}\right\|_{\mathcal{H}^{1}(\Omega)} \leq C h$.

La discrétisation du problème $\{(13),(14)\}$ fait apparaître, pour un élément de longueur $h$ (pour $E I$ et $f$ constants), les matrices suivantes

$$
\begin{aligned}
& \frac{h}{6 E I}\left[\begin{array}{cc}
2 & 1 \\
1 & 2
\end{array}\right]\left[\begin{array}{l}
M_{1} \\
M_{2}
\end{array}\right]+\frac{1}{h}\left[\begin{array}{cc}
1 & -1 \\
-1 & 1
\end{array}\right]\left[\begin{array}{l}
u_{1} \\
u_{2}
\end{array}\right]=0, \\
& -\frac{1}{h}\left[\begin{array}{cc}
1 & -1 \\
-1 & 1
\end{array}\right]\left[\begin{array}{l}
M_{1} \\
M_{2}
\end{array}\right]+\frac{\alpha h}{6}\left[\begin{array}{ll}
2 & 1 \\
1 & 2
\end{array}\right]\left[\begin{array}{l}
u_{1} \\
u_{2}
\end{array}\right] \\
& -\frac{P}{h}\left[\begin{array}{cc}
1 & -1 \\
-1 & 1
\end{array}\right]\left[\begin{array}{l}
u_{1} \\
u_{2}
\end{array}\right]=f\left[\begin{array}{l}
h / 2 \\
h / 2
\end{array}\right] .
\end{aligned}
$$

On effectue l'assemblage pour les $N$ éléments. On notera

$$
\Sigma^{\mathrm{T}}=\left[M_{1}, M_{2}, \ldots, M_{N}, M_{N+1}\right] \text { et } D^{\mathrm{T}}=\left[u_{1}, u_{2}, \ldots, u_{N}\right]
$$

d'où la forme matricielle

$$
\left[\begin{array}{cc}
S & B \\
B^{\mathrm{T}} & C
\end{array}\right]\left[\begin{array}{c}
M \\
D
\end{array}\right]=\left[\begin{array}{l}
O \\
F
\end{array}\right]
$$

On présente divers exemples et notamment une solution analytique qui est comparée à la solution numérique

$$
\alpha=4.5, l=1, P=5, E I=0.5, f=1 \text {. }
$$

On calcule l'erreur

$$
\Delta=\int_{0}^{l}\left(u-u_{h}\right)^{2} \mathrm{~d} \Omega .
$$

On obtient $\Delta=1.38 \times 10^{-5}$ pour le nombre d'èlements $n=6$ et $\Delta=2.62 \times 10^{-5}$ pour $n=32$; les traces des moments (Fig. 1) et des deplacements (Fig. 2) sont preséntés.

Le système a été inversé par une méthode de Gauss et, dès qu'on atteint plus de 30 éléments, les erreurs sont stationnaires. 


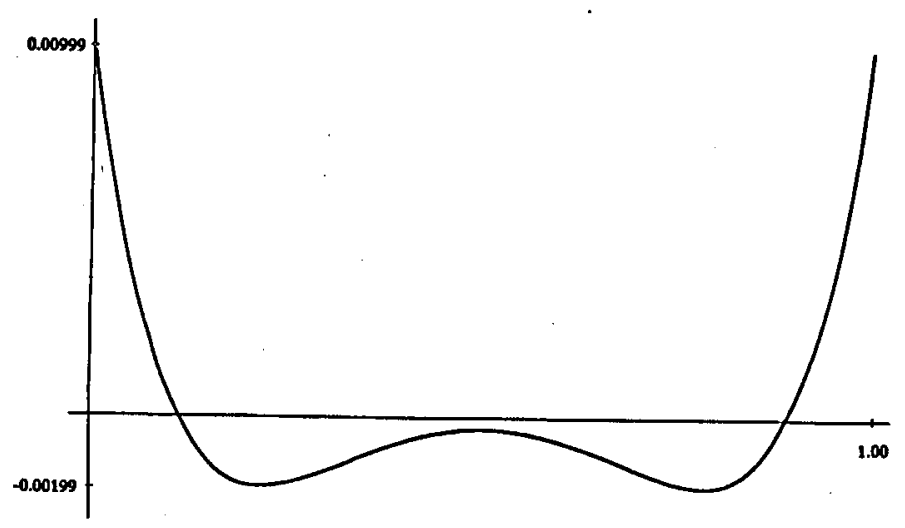

Fig. 1. Trace des moments: $\alpha=5000.0000$, nombre d'elements $=32$, prècontrainte $=-5.0$, charge $f=1.0000$, produit $E I=0.5000$.

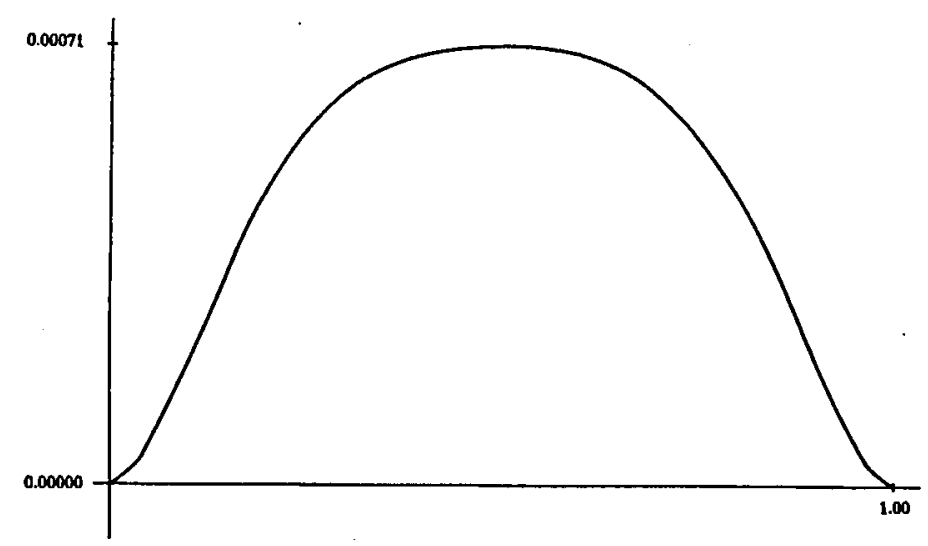

Fig. 2. Trace des deplacements: $\alpha=5000.0000$, nombre d'elements $=32$, prècontrainte $=-5.0$, charge $f=1.0000$, produit $E I=0.5000$.

Le cas particulier intéressant est un calcul de couche fine et où existe des effets de couche limite notables auprès des encastrements. L'exemple présenté fait apparaître un gradient de moment important. Il traduit l'influence d'un effort tranchant. Cet exemple montre aussi que, loin des encastrements, la poutre fonctionne comme une membrane mince.

Cet exemple est le fruit d'un travail effectué par les élèves de l'E.N.I.S.E. de 5ème année, auquel de Universitaire polonais de Lódź ont participé, dans le cadre des échanges Franco-Polonais.

\section{Références}

[1] C. Surry, Thése d'Etat, Université Rennes-1, 1982. 